\title{
Mitochondrial dysregulation and glycolytic insufficiency functionally impair CD8 T cells infiltrating human renal cell carcinoma
}

Peter J. Siska, ${ }^{1,2,3}$ Kathryn E. Beckermann, ${ }^{1,3,4}$ Frank M. Mason, ${ }^{3,4}$ Gabriela Andrejeva, ${ }^{1,3}$ Allison R. Greenplate, ${ }^{1,3,5}$ Adam B. Sendor, ${ }^{6}$ Yun-Chen J. Chiang, ${ }^{6}$ Armando L. Corona, ${ }^{6}$ Lelisa F. Gemta, ${ }^{7}$ Benjamin C. Vincent, ${ }^{6}$ Richard C. Wang, ${ }^{8}$ Bumki Kim, ${ }^{9}$ Jiyong Hong, ${ }^{9}$

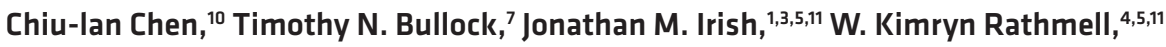
and Jeffrey C. Rathmell1,3,5,11

'Department of Pathology, Microbiology, and Immunology, Vanderbilt University Medical Center, Nashville, Tennessee, USA. ${ }^{2}$ Department of Internal Medicine III, University Hospital Regensburg, Regensburg, Germany. ${ }^{3}$ Vanderbilt Center for Immunobiology, Vanderbilt University School of Medicine, Nashville, Tennessee, USA. ${ }^{4}$ Department of Medicine, Division of Hematology and Oncology, Vanderbilt University Medical Center, Nashville, Tennessee, USA. ${ }^{5}$ Department of Cancer Biology, Vanderbilt University, Nashville, Tennessee, USA. ${ }^{6}$ Lineberger Cancer Center, University of North Carolina at Chapel Hill, Chapel Hill, North Carolina, USA. 'Department of Pathology, University of Virginia, Charlottesville, Virginia, USA. ${ }^{8}$ Department of Dermatology, UT Southwestern Medical Center, Dallas, Texas, USA. ${ }^{9}$ Department of Chemistry, Duke University, Durham, North Carolina, USA. ${ }^{10}$ Department of Biostatistics, ${ }^{11}$ Vanderbilt-Ingram Cancer Center, Vanderbilt University Medical Center, Nashville, Tennessee, USA.

Cancer cells can inhibit effector T cells (Teff) through both immunomodulatory receptors and the impact of cancer metabolism on the tumor microenvironment. Indeed, Teff require high rates of glucose metabolism, and consumption of essential nutrients or generation of waste products by tumor cells may impede essential T cell metabolic pathways. Clear cell renal cell carcinoma (ccRCC) is characterized by loss of the tumor suppressor von Hippel-Lindau (VHL) and altered cancer cell metabolism. Here, we assessed how ccRCC influences the metabolism and activation of primary patient ccRCC tumor infiltrating lymphocytes (TIL). CD8 TIL were abundant in ccRCC, but they were phenotypically distinct and both functionally and metabolically impaired. cCRCC CD8 TIL were unable to efficiently uptake glucose or perform glycolysis and had small, fragmented mitochondria that were hyperpolarized and generated large amounts of ROS. Elevated ROS was associated with downregulated mitochondrial SOD2. CD8 T cells with hyperpolarized mitochondria were also visible in the blood of ccRCC patients. Importantly, provision of pyruvate to bypass glycolytic defects or scavengers to neutralize mitochondrial ROS could partially restore TIL activation. Thus, strategies to improve metabolic function of CCRCC CD8 TIL may promote the immune response to cCRCC.

Authorship note: WKR and JR are co-corresponding authors to this work.

Conflict of interest: The authors have declared that no conflict of interest exists.

Submitted: February 13, 2017

Accepted: May 5, 2017

Published: June 15, 2017

Reference information: JCI Insight. 2017;2(12):e93411. https:// doi.org/10.1172/jci.insight.93411

\section{Introduction}

It has long been a goal to activate the adaptive immune system to eliminate cancer cells and tumors. Prior approaches to solely immunize with cancer cells or tumor-specific antigens, however, have yielded disappointing results (1). Nevertheless, tumors can be highly infiltrated with immune cells, including both lymphocytes and myeloid cells, and the degree and location of infiltrating immune cells can be predictive of outcome $(2,3)$. It is now apparent that the tumor microenvironment, influenced by cancer cells, can convey immunomodulatory signals to directly inhibit immune responses and prevent or impair antitumor immunity $(4,5)$. Blockade of these immune-inhibitory signals or stimulation of immune-activating receptors now offers the potential to reactivate the immune system and allows tumor-reactive $\mathrm{T}$ cells to seek out and eliminate cancer cells. In particular, blockade of interactions of the $\mathrm{T}$ cell inhibitory receptor programed death-1 (PD-1) with its ligands (PD-L1 and PD-L2) have shown tremendous promise to efficiently target a variety of cancers $(4,5)$. Clear cell renal cell carcinoma (ccRCC) is responsive to both high-dose IL-2 
and PD-1 checkpoint blockade (6-8). However, these immune therapies are effective in only a portion of patients, and duration of response can be limited. It is important to determine how additional interactions of $\mathrm{T}$ cells with the tumor microenvironment may suppress antitumor immunity.

One potential barrier to T cell-mediated elimination of cancer cells is the requirement of lymphocytes to undergo metabolic reprogramming and induce high rates of aerobic glycolysis for effector function (9-11). Resting T cells utilize a catabolic and largely oxidative metabolism that promotes survival and quiescence. Upon activation, however, $\mathrm{T}$ cells upregulate nutrient transporters, including the glucose transporter Glut1, and sharply increase anabolic glucose and amino acid metabolism. In addition to increased glycolysis, mitochondria are also dynamically remodeled upon $\mathrm{T}$ cell activation and undergo a process of fragmentation, and cristae become condensed (12). While this mitochondrial structure can impair the efficiency of electron transport (13), mitochondria nevertheless continue to play essential roles in $\mathrm{T}$ cell activation. While excessive mitochondrial ROS can lead to an adaptive stress response, ROS can also regulate signaling (14). Mitochondria are also critical to produce biosynthetic intermediates and ATP $(12,14)$. Failure to induce these metabolic transitions can impair immune activity, as Glut1 deficiency, impaired mitochondrial electron transport, insufficient nutrient availability, or metabolic inhibition can prevent proliferation, T cell activation, and effector function $(9,14-17)$.

Cancer cells may inhibit $\mathrm{T}$ cell metabolic reprogramming to impair antitumor immunity through both direct signaling and indirect effects, altering the local composition of nutrients and waste products present in the microenvironment. PD-1 ligation can suppress $\mathrm{T}$ cell signaling through phospatidylinositide-3-kinase (PI3K)/Akt/mTOR complex 1, leading to reduced glucose uptake and aerobic glycolysis (18-22) and promotion of lipid oxidation $(19,23)$. In addition, consumption of nutrients by cancer cells may limit $\mathrm{T}$ cell access to glucose and key amino acids needed for $\mathrm{T}$ cell activation and effector signaling pathways $(10,24,25)$. Tumor cell production of lactic acid can also suppress $\mathrm{T}$ cell signaling through NFAT and IFN- $\gamma$ secretion to impair antitumor immunity (11). Tumor infiltrating lymphocytes (TIL) have been reported to lose mitochondrial mass in a melanoma model, rendering $\mathrm{T}$ cells unable to efficiently meet bioenergetic needs (26). While restoring $\mathrm{T}$ cell glucose uptake and glycolytic intermediates $(24,27)$ or mitochondrial mass $(26)$ can improve TIL function, the mechanisms and role of $\mathrm{T}$ cell metabolic inhibition in cancer remain poorly understood.

ccRCC is highly associated with mutations of the von Hippel-Lindau $(V H L)$ gene $(28,29)$. The $V H L$ mutation permits stabilization of hypoxia inducible factors (HIF-1 $\alpha$ and HIF-2 $\alpha$ ), transcription factors that promote tumor cell glycolysis, and altered metabolism through altered transcriptional programs. This altered metabolic environment may influence outcomes following PD-1 blockade therapy (30). Here, we assessed the functionality and cell intrinsic metabolism of ccRCC TIL from 54 patients. While CD4 ccRCC TIL were only moderately affected, CD8 ccRCC TIL activated poorly and failed to proliferate upon stimulation. Although CD8 ccRCC TIL expressed Glut1 and maintained mitochondrial mass, TCR stimulation failed to induce glucose uptake, and mitochondria appeared fragmented. Mitochondria were also hyperpolarized and produced high levels of ROS. Importantly, ccRCC CD8 TIL activation was improved by addition of pyruvate or mitochondrial ROS scavengers. Together, these data show that metabolic adaptation and impairments of resident ccRCC TIL contribute to poor $\mathrm{T}$ cell function and that restoring $\mathrm{T}$ cell metabolism may improve functionality of T cells in the ccRCC tumor microenvironment.

\section{Results}

To identify barriers to antitumor immunity in ccRCC, we examined T cells from 54 freshly resected patient tumors. Consistent with findings in The Cancer Genome Atlas (TCGA) (29) (Figure 1A) in which ccRCC had the highest $C D 8$ a signature of all nonlymphoid solid tumor types, CD8 T cells were found to be abundant in immunofluorescence analysis of ccRCC tissue (Figure 1B). Fresh tumor sections were then dissociated into single cell suspensions to examine cell surface marker expression. Several T cell activation and exhaustion markers were coordinately upregulated on ccRCC CD8 TIL, most prominently CD69 and PD-1 (Figure 1C). While additional inhibitory receptors may also play a role in ccRCC, expression of Tim3, CTLA4, and LAG-3 was not found to be significantly elevated.

To further phenotype ccRCC TIL, we examined resting or stimulated peripheral blood mononuclear cells (PBMC) and primary ccRCC tumor tissue using high-dimensional mass cytometry. SPADE analyses cluster phenotypically-like cell populations, and they showed that activated healthy donor PBMC CD8 $\mathrm{T}$ cells underwent a phenotypic transition to increase expression of CD27, CD38, and PD-1 (Figure 1A 
A

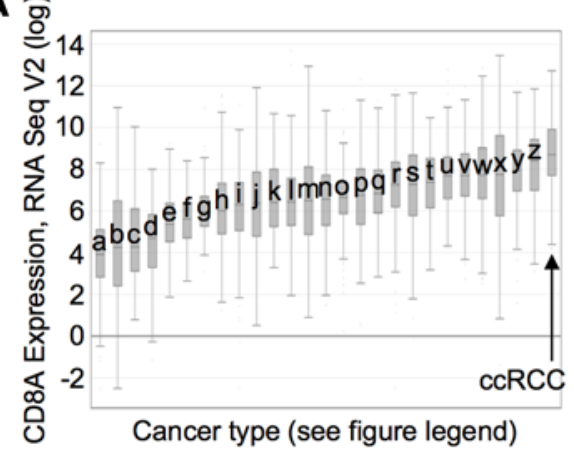

B

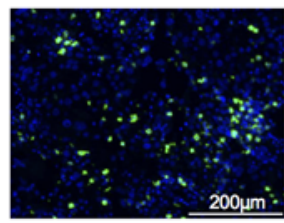

DAPI

CD8
C

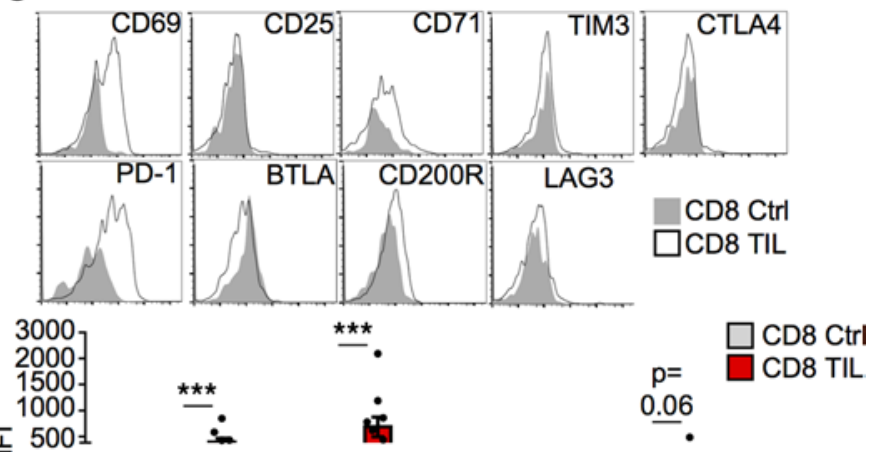

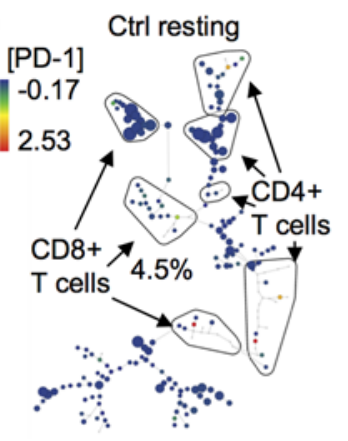

Ctrl anti-CD3 stim.

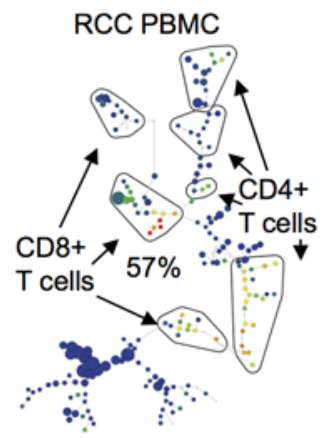

RCC TIL
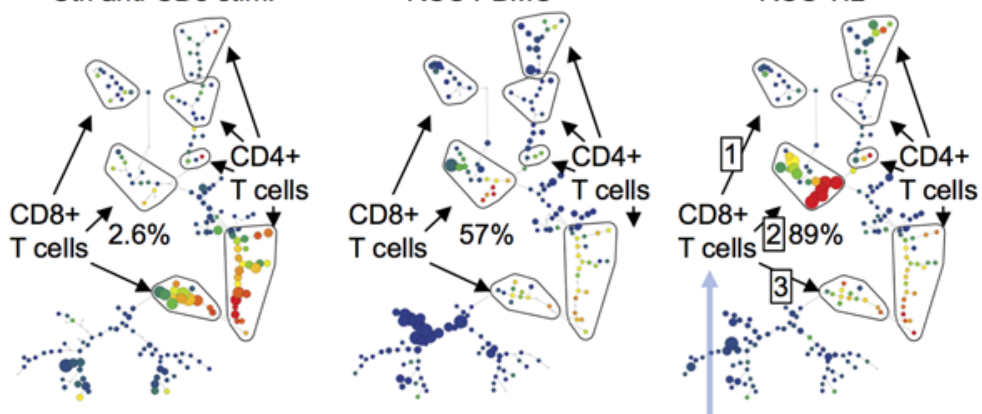

\begin{tabular}{|l|}
\hline 1 \\
\hline 2 \\
\hline 3 \\
\hline
\end{tabular}

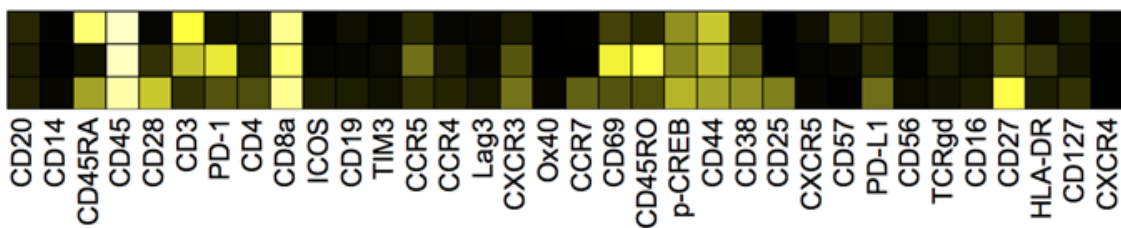

Figure 1. Dissecting the phenotype of ccRCC CD8 TIL. (A) CD8 expression in nonlymphoid solid tumors queried in The Cancer Genome Atlas. a, glioma; b, uveal melanoma; c, adenoid cystic carcinoma (Ca); d, chromophobe renal cell carcinoma (cRCC); e, glioblastoma; f, pheochromocytoma and paraganglioma; g, uterine carcinosarcoma; h, liver Ca; i, colorectal Ca; j, bladder Ca; k, cholangiocarcinoma; l, papillary RCC; m, sarcoma; n, ovarian Ca; o, prostate Ca; p, uterine Ca; q, thyroid Ca; r, breast Ca; s, head and neck Ca; t, pancreas Ca; u, mesothelioma; v, lung sq Ca; w, cervical Ca; $x$, melanoma; , lung adeno $\mathrm{Ca}$; $z$, testicular tumors. (B) Representative IHC staining of 16 RCC patient sections for CD8 and DAPI. (C) Expression of selected markers on CD8 T cells from RCC patients $(n=5-10)$ or healthy donors $(n=12-17)$ measured with flow cytometry. Error bars represent $\pm \mathrm{SEM} ;{ }^{*} P<0.05,{ }^{* *} P<0.01$, and ${ }^{* * *} P<0.001$, unpaired Student's $t$ test. (D) Mass cytometric analysis of TIL and PMBC from RCC patients $(n=3)$ or resting and anti-CD3 stimulated PBMC from healthy donors $(n=2)$ using selected markers. Unsupervised clustering and SPADE diagram visualization was performed using Cytobank software. Heatmap shows relative expression of selected markers on 3 distinct CD8 T cell subpopulations from a representative RCC patient sample. Percentages indicate relative abundance of Group 2 in all CD8 T cells

and Supplemental Figure 1, A-C; supplemental material available online with this article; https://doi. org/10.1172/jci.insight.93411DS1). Interestingly, ccRCC CD8 T cells were phenotypically distinct from both resting and acutely activated healthy donor CD8 T cells. Similar to previous studies (31), the majority of ccRCC CD8 TIL were $\mathrm{CD} 69^{\text {high }} \mathrm{CD} 45 \mathrm{RO}^{+} \mathrm{PD}-1^{\text {high }} \mathrm{CD} 27^{\text {low }} \mathrm{CD} 38^{\text {low }}$ (population no. 2), suggesting an effector memory-like $\mathrm{T}$ cell phenotype. Further comparison of PD-1 $1^{\text {high }}$ and $\mathrm{PD}-1^{\text {low }} \mathrm{CD} 8^{+} \mathrm{T}$ cells showed that PD-1 $1^{\text {high }} \mathrm{CD} 8^{+}$TIL were selectively enriched for expression of CD69, CD45RO, CD38, and CD27, while having lower CD44 expression (Supplemental Figure 1D). ccRCC CD8 TIL were enriched in effector and central memory phenotype cells (Supplemental Figure 2). Interestingly, ccRCC patient PBMC contained a similarly enriched population of CD8 $\mathrm{T}$ cells.

Because ccRCC CD8 TIL can be functionally impaired (32), single cell suspensions of ccRCC tumors were stimulated in vitro to assess TIL activation. ccRCC CD8 TIL showed reduced capability to upregulate 

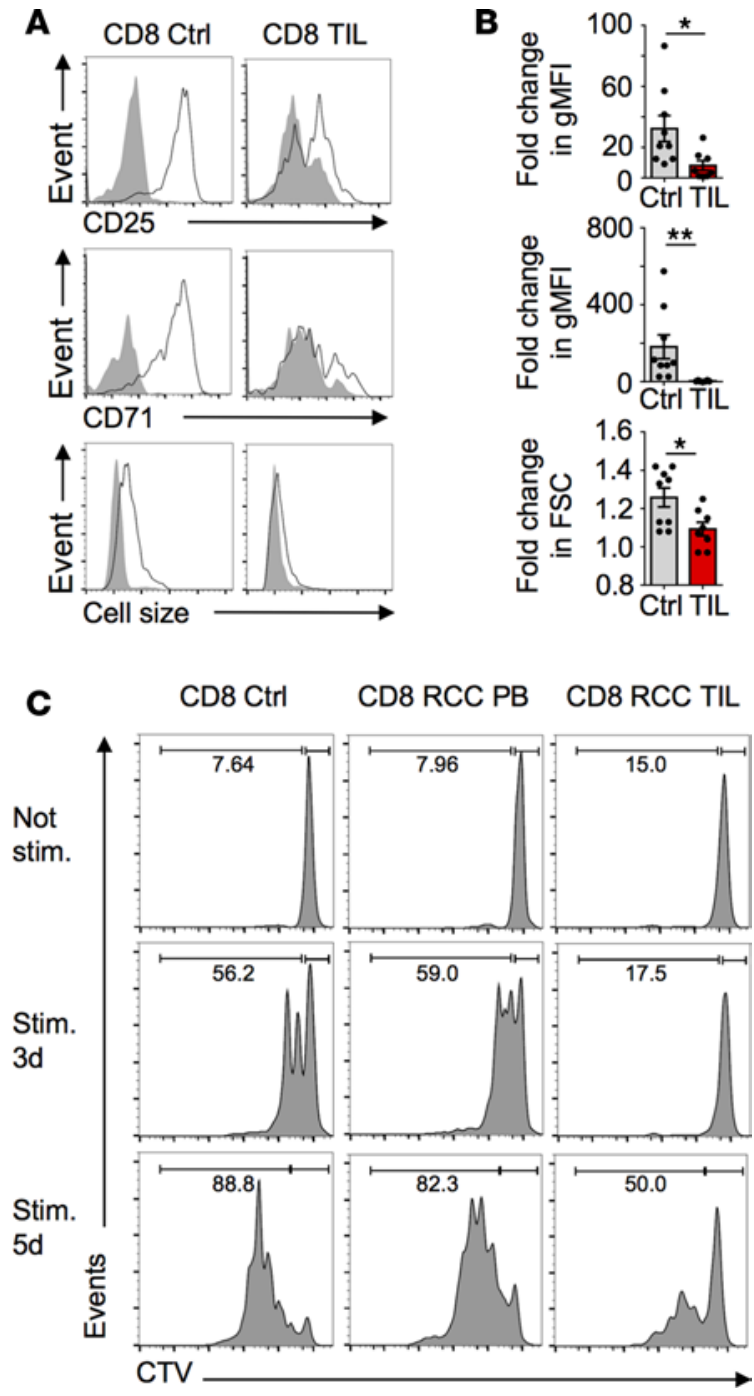

Figure 2. Impaired activation and proliferation of cCRCC CD8 TIL. (A and B) RCC samples $(n=6-8)$ and controls $(n=9)$ were in vitro anti-CD3-stimulated for 3 days, and expression of CD25 and CD71 and cell size of CD8 T cells were measured with flow cytometry. Data comparing relative increase in activation were analyzed using Wilcoxon rank sum test with continuity correction. ${ }^{*} P<0.05$ and ${ }^{* *} P<0.01$. (C) Cells from healthy donors, RCC patient blood, or RCC patient tumors were stimulated as in $\mathbf{A}$ and $\mathbf{B}$, and CD8 T cell proliferation was measured after 3 and 5 days using CellTrace Violet. Data are representative of 3 controls and 4 RCC patient samples.

$\mathrm{T}$ cell activation markers and had impaired cell growth and limited proliferation relative to healthy donor or ccRCC patient CD8 PBMC (Figure 2, A-C). ccRCC CD4 TIL, in contrast, showed only minor impairments (Supplemental Figure 3, A and B). Defects were not due to continuous inhibitory PD-1 signaling, as addition of PD-1 blocking antibody to in vitro cultures was not sufficient to alter activation or cell growth (Supplemental Figure 3C).

$\mathrm{T}$ cells require metabolic reprogramming and increased aerobic glycolysis to gain effector function $(9,15,22,33)$. While tumor cells can deplete nutrients $(10,15,24,34)$, no decrease in interstitial glucose levels was observed in ccRCC tumors relative to adjacent normal kidney tissue (Figure 3A). Nevertheless, lactate can inhibit $\mathrm{T}$ cell function $(11,35)$ and was increased in ccRCC interstitial fluid. ccRCC CD8 TIL had normal or a trend toward increased expression of glucose transporter Glut1 and hexokinase 2 (HK2) (Figure 3B). The mTORC1 pathway regulates T cell metabolism (22) and appeared to be activated, as the downstream mTORC1 effector phosphor-S6 was significantly elevated (Figure 3B). Inhibition of this pathway by rapamycin decreased CD8 $\mathrm{T}$ cell upregulation of CD25 and CD71 following stimulation (Figure 3C), demonstrating an essential role for mTORC1 in ccRCC CD8 TIL.

Efficient glucose metabolism promotes CD8 $\mathrm{T}$ cell effector function (36) and can enhance production of memory CD8 cells (37). Nevertheless, cCRCC CD8 TIL had defects in both glucose uptake and glycolysis. Although cCRCC CD8 TIL had an activated phenotype and total cellular Glut1 and HK2 expression appeared normal (Figure 3B), basal uptake of the fluorescent glucose analog 2NBDG was similar to resting control CD8 T cells (Supplemental Figure 4A). In addition, ccRCC CD8 TIL were unable to increase glucose uptake following stimulation (Figure 4A). In contrast, ccRCC CD4 TIL remained able to increase glucose uptake upon stimulation (Supplemental Figure 4B). Phosphorylation of Glut1 S226 by protein kinase C (PKC) may promote Glut1 cell surface localization and activity (38). However, we found ccRCC CD8 TIL unable to increase Glut1 phosphorylation (Figure 4B), potentially rendering this transporter unable to access extracellular glucose. In addition, ccRCC CD8 TIL showed glycolytic defects downstream of glucose uptake, as expression of GAPDH was significantly lower (Figure 4C). Consistent with a better ability to maintain glucose uptake and metabolism, ccRCC CD4 TIL maintained GAPDH expression (Supplemental Figure 4C). Importantly, ccRCC CD8 TIL appeared adapted to having a lower dependence on glucose and glycolysis, and activation was not further suppressed in low glucose conditions, as it was for control CD8 (Figure 4D).

Mitochondria can efficiently provide energy from pyruvate and could compensate for impaired glycolysis of CD8 TIL, but have been reported to be depleted in melanoma and head-and-neck-cancer TIL (26). Reduced electron transport has also been associated with effector CD8 T cells (12), and T cells with low mitochondrial membrane potential can have enhanced antitumor function (39), suggesting that mitochondria may play critical roles in antitumor immunity. We, therefore, assessed mitochondria in control and ccRCC CD8 TIL. Interestingly, mitochondria in control CD8 T cells appeared in fused aggregates (Figure 5A, and Supplemental Videos 1 and 2) while mitochondria in ccRCC CD8 TIL appeared small and dispersed (Figure 5A and Supplemental Videos 3-5) when analyzed using super-resolution confocal microscopy. Altered cellular metabolism can impact mitochondrial dynamics and lead to mitochondrial fragmentation and dysfunction $(12,40,41)$. Given the apparent altered size and distribution of mitochondria in cCRCC CD8 TIL, electron microscopy (EM) was next used to better assess mitochondrial structure. 
A

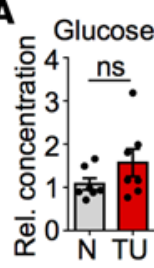

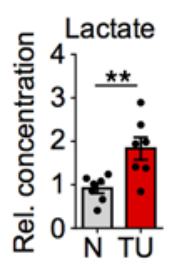

C

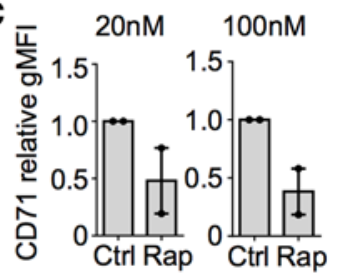

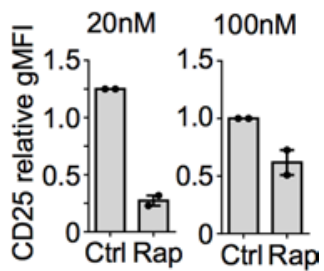

B

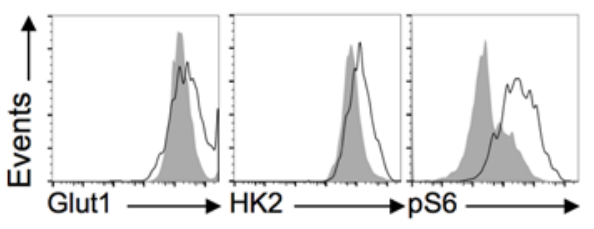

CD8 Ctrl CD8 TIL

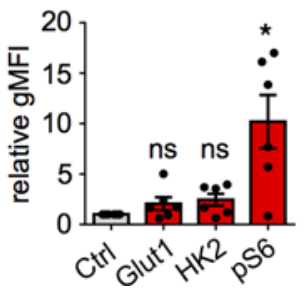

Figure 3. ccRCC CD8 TIL express Glut1 and HK2 and show induction of mTORC1. (A) Clucose and lactate concentrations were measured by NMR in interstitial fluid extracts from tumor tissue (TU) and surrounding normal kidney tissue (N) on 7 RCC patients. Concentrations were normalized to mean concentration of N. (B) Expression of Glut1 and HK2 and phosphorylation of S6 was measured on 6 RCC samples and 12 controls. (C) RCC samples $(n=2)$ were treated over-night with control or rapamycin in selected concentrations, and CD25 and CD71 expression was measured after drug wash-out followed by stimulation for 3 days. ${ }^{*} P<0.05,{ }^{* *} P<0.01$ Student's $t$ test.

Consistent with small mitochondria in confocal images, EM demonstrated that mitochondria from ccRCC CD8 TIL were predominantly small and round, rather than large rod shapes found in control CD8 T cells (Figure 5, B and C). Further, mitochondria in ccRCC CD8 TIL appeared predominantly in the condensed configuration relative to the more orthodox configuration of control CD8 $\mathrm{T}$ cells.

Despite apparent mitochondrial fragmentation and altered structure, mitochondrial mass was not decreased. MitoTracker Green (MTG), which indicates mitochondrial inner membrane mass irrespective of membrane potential, was increased rather than decreased in ccRCC CD8 TIL (Figure 6A). MTG staining of peripheral blood CD8 $\mathrm{T}$ cells from healthy and ccRCC donors, however, was equivalent (Supplemental Figure 5A). Expression of multiple mitochondrial electron transport proteins in ccRCC CD8 TIL was normal and coordinated (Supplemental Figure 5, B and C), suggesting a dysregulation of mitochondrial morphology irrespective of mitochondrial protein expression. PGC1 $\alpha$ promotes mitochondrial biogenesis, and deficiency of this transcription factor may decrease mitochondrial content and expression of electron transport components in T cells $(20,26,42)$. However, PGC1 $\alpha$ expression appeared normal in ccRCC CD8 TIL (Supplemental Figure 5D). Mitochondrial metabolism remained important for ccRCC CD8 TIL, as inhibition of electron transport complex I with metformin or phenformin reduced ccRCC CD8 TIL in vitro survival (Supplemental Figure 5E).

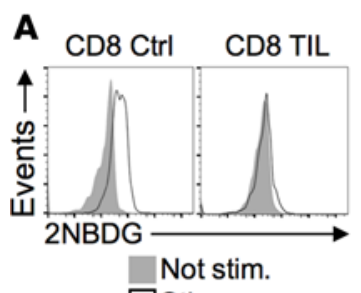

$\square$ Stim.

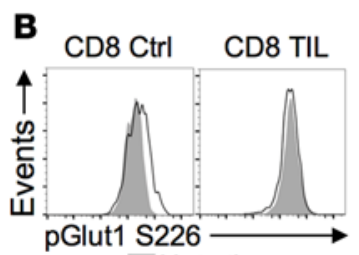

Not stim.

J.im.
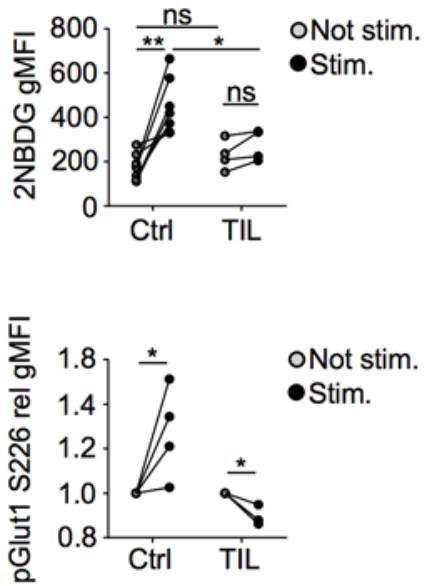

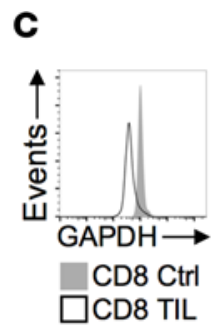

D
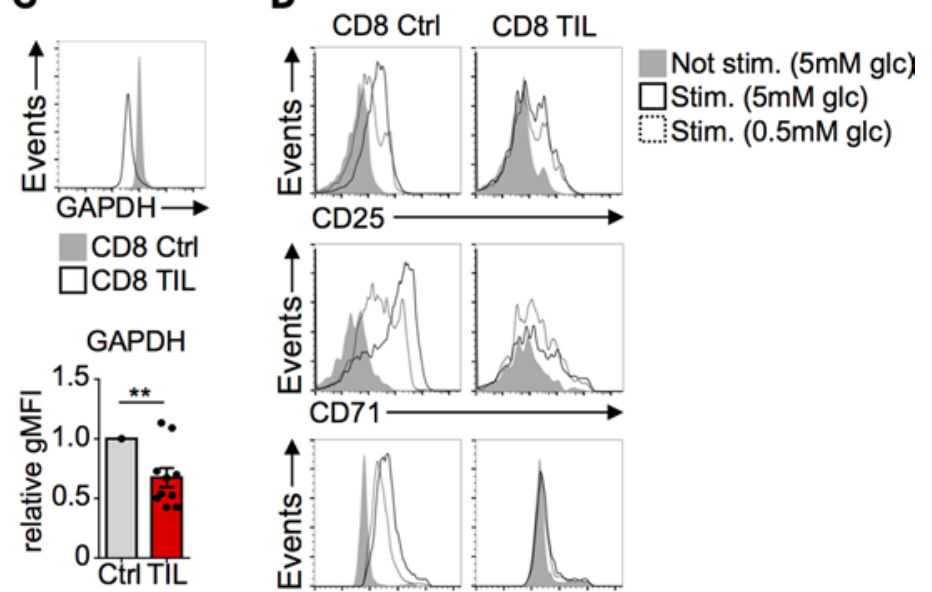

Figure 4. Decreased glucose uptake and metabolism of ccRCC CD8 TIL correlates with lower glucose dependency. (A) 2NBDC uptake of CD8 Ctrl ( $n=7$ ) and CD8 TIL $(n=4)$ after 3 days of stimulation. (B) Cells were stimulated as in A, and Glut1 phosphorylation at S226 was measured with flow cytometry. (C) GAPDH expression measured on $10 \mathrm{RCC}$ patient samples and 14 controls. Geometric mean fluorescence intensity (gMFI) values were normalized to controls for each separate experiment. (D) Cells were stimulated for 3 days in media containing indicated glucose concentrations, and expression of CD25 and CD71 and cell size was measured with flow cytometry. Data are representative of 4 independent experiments. Error bars represent \pm SEM; ${ }^{*} P<0.05$, ${ }^{* *} P<0.01$ and ${ }^{* *} P<0.001$, paired Student's $t$ test. 
A
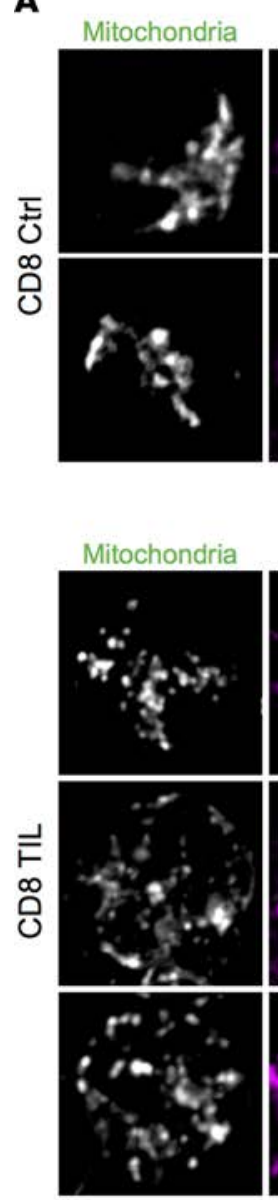

CD8

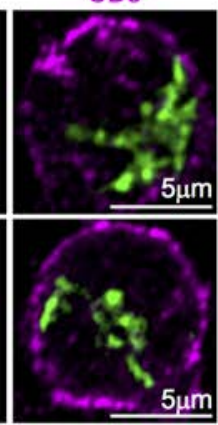

DAPI

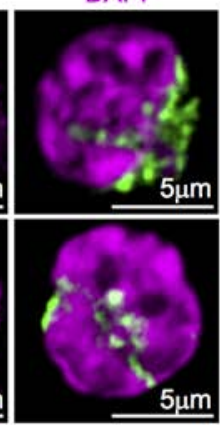

Mitochondria

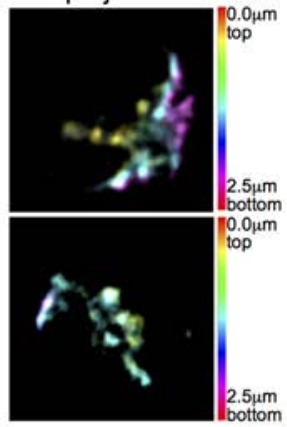

Mitochondria
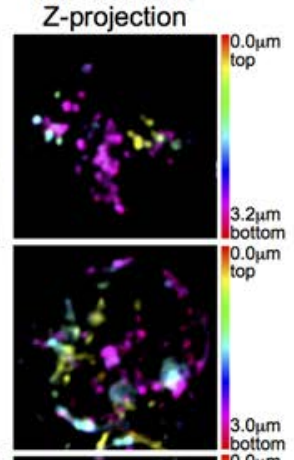
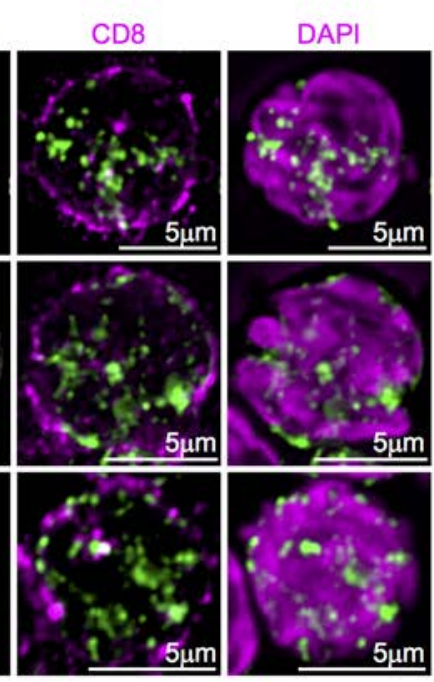

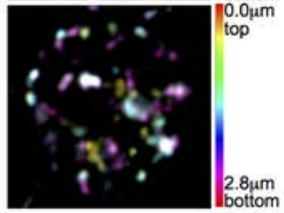

B

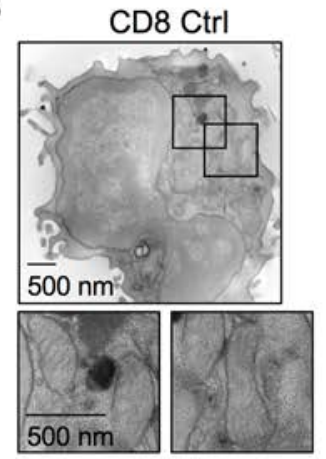

CD8 Ctrl

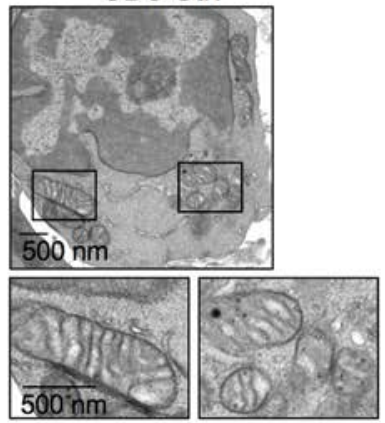

C

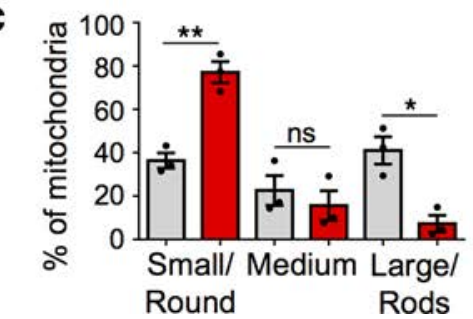

CD8 TIL

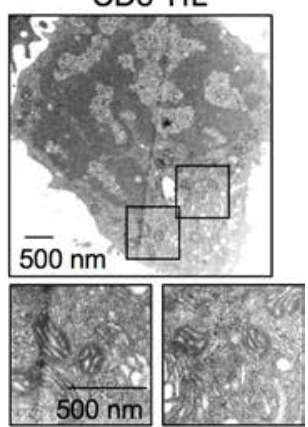

CD8 TIL

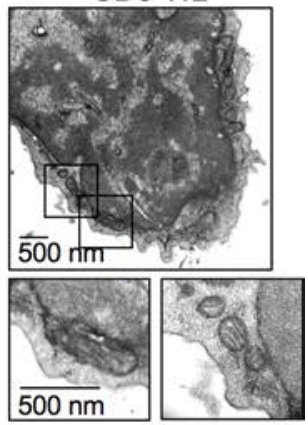

CD8 Ctrl CD8 TIL

Figure 5. Dysregulation of mitochondrial morphology in ccRCC CD8 TIL. (A) Confocal microscopy of healthy control CD8 T cells (control, $n=2$ donors, 52-105 cells per donor) or CD8 ccRCC TIL (TIL, $n=2$ patients, 35-118 cells per patient), stained for mitochondria (Mitotracker), CD8, and DAPI. At right, mitochondria are color-coded based on z-position. Scale bars: $5 \mu \mathrm{m}$. (B and C) Electron microscopy was performed on RCC patient samples $(n=3)$ and healthy donors $(n=3)$. Shown are images of 2 patient samples and 2 controls. Mitochondrial morphology was assessed on $16-20$ CD8 T cells from each sample. Scale bars: $500 \mathrm{~nm}$. Error bars represent $\pm \mathrm{SEM} ;{ }^{*} P<0.05,{ }^{* *} P<0.01$, Student's $t$ test.

Altered structure and maintenance, along with increased mitochondrial mass, suggested distinct mitochondrial function in ccRCC CD8 TIL compared with control CD8 T cells. Indeed, a greater proportion of ccRCC CD8 TIL had hyperpolarized mitochondria than control CD8 T cells from healthy donors (Figure 6B). Hyperpolarized mitochondria can be a source of ROS in macrophages (43), and ccRCC CD8 TIL also had high levels of mitochondrial ROS (Figure 6C). Total cellular or cytoplasmic ROS, however, were decreased in ccRCC CD8 TIL relative to control T cells (Figure 6D). An adaptive cytosolic mechanism to neutralize ROS is increased cystine uptake and glutathione production. Using a fluorescent indicator (44), we found cystine uptake to be increased in cCRCC CD8 TIL (Figure 6E). This was most pronounced in ccRCC CD8 TIL with elevated mitochondrial ROS (Figure 6F). Peripheral blood T cells from healthy and ccRCC donors were, however, equivalent for both total cellular ROS and cystine uptake (Supplemental Figure 6, A and B). After uptake, cystine is converted to cysteine, and ccRCC CD8 TIL also had elevated intracellular levels of cysteine using a distinct dye indicator (Supplemental Figure 6C). Glutathione is generated from cysteine to neutralize ROS and was also increased in ccRCC CD8 TIL (Supplemental Figure 6D). Increased ROS may cause DNA damage in T cells (39), and although ATM phosphorylation was not altered, ccRCC $\mathrm{CD} 8 \mathrm{TIL}$ had increased levels of the DNA damage-indicator $\gamma \mathrm{H} 2 \mathrm{AX}$ (Supplemental Figure 6E).

Increased mitochondrial ROS as a consequence of altered ccRCC CD8 TIL mitochondrial dynamics and function may also impair T cell function. SOD1 and -2 play roles to neutralize cytoplasmic and mitochondrial ROS, respectively (45). SOD1 levels were unchanged in ccRCC CD8 TIL (Supplemental Figure 7, A and B). 
A
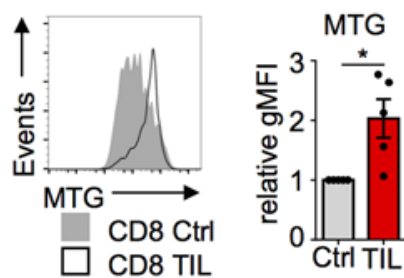

B
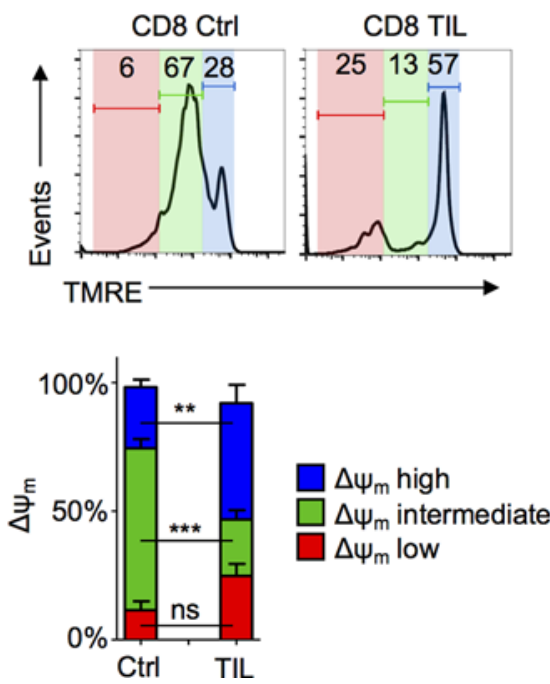

C

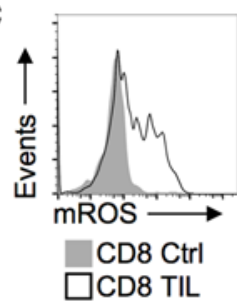

D

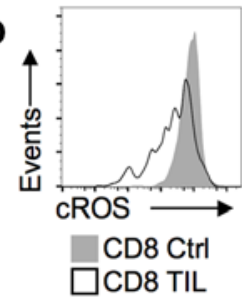

G
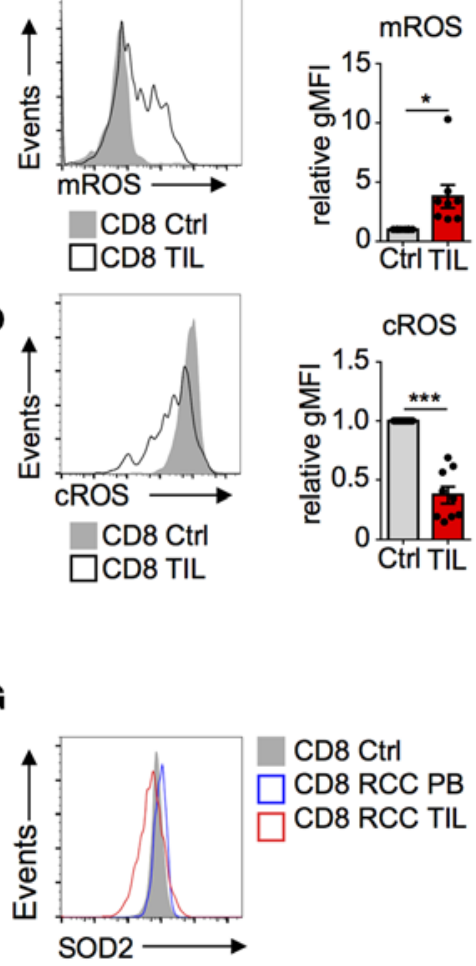

E
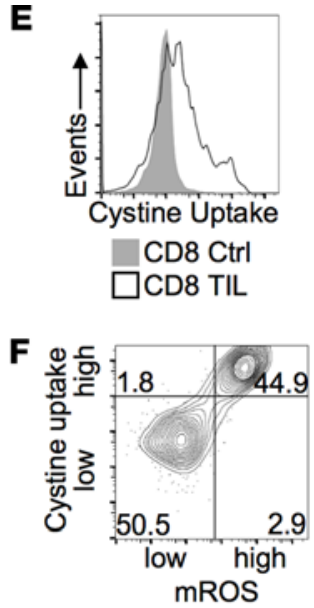

CystineFITC high
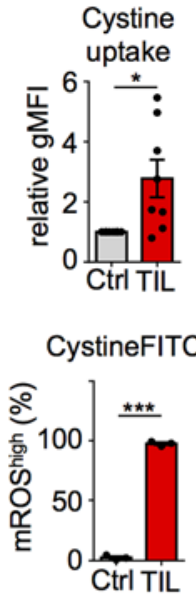

Figure 6. ccRCC CD8 TIL mitochondrial hyperpolarization and ROS accumulation correlate with increased uptake of cystine. (A) Mitochondrial mass/ mitochondrial membrane content was measured with MitoTracker Green, which is independent of membrane potential $(n=5$ RCC patients and $n=8$ healthy donors). (B) Mitochondrial membrane potential measurements were performed using TMRE on 4 RCC and 10 control samples. (C and D) Mitochondrial and cytosolic ROS were measured on RCC patient samples using MitoSOX $(n=8)$ and DCFDA $(n=9)$, respectively. (E) Uptake of cystine was performed using CystineFITC on CD8 T cells from RCC patients $(n=8)$ and controls $(n=12)$. (F) Mitochondrial ROS and cystine uptake was measured on CD8 T cells with flow cytometry. (C) Expression of SOD2 was measured on CD8 T cells from controls, RCC patient blood, and RCC patient TIL. Error bars represent \pm SEM; ${ }^{*} P<0.05,{ }^{* *} P<0.01$, and ${ }^{* * *} P<0.001$, Student's $t$ test.

In contrast, while SOD2 levels were equivalent between peripheral blood of healthy and ccRCC donors, expression of this protein was downregulated in CD8 TIL in the majority of ccRCC tumors (Figure 6G and Supplemental Figure 7B). ccRCC CD4 TIL, in contrast, largely retained SOD2 (Supplemental Figure 7C).

Tumor-experienced $\mathrm{T}$ cells can be found in the periphery of cancer patients $(46,47)$. While the percent of $\mathrm{TMRE}^{\text {high }} \mathrm{CD}^{+} \mathrm{T}$ cells was similar in the blood of healthy and ccRCC donors (Supplemental Figure 8A), $\mathrm{PD}-\mathrm{1}^{\text {high }} \mathrm{CD} 8^{+} \mathrm{T}$ cells with hyperpolarized mitochondria were detectable in peripheral blood from ccRCC patients (Figure 7A). Further, these cells also showed increased mitochondrial ROS and MTG staining, while cytoplasmic ROS was decreased relative to PBMC PD- $1^{\text {low }} \mathrm{CD} 8^{+} \mathrm{T}$ cells (Supplemental Figure 8B). Patient PBMC $\mathrm{PD}-1^{\text {high }} \mathrm{CD} 8^{+} \mathrm{T}$ cells showed higher levels of mitochondrial polarization than $\mathrm{PD}-1^{\text {low }} \mathrm{CD} 8^{+} \mathrm{T}$ cells (Supplemental Figure 8C). Importantly, mitochondrial polarization was greater in PBMC PD- $1^{\text {high }} \mathrm{CD} 8^{+} \mathrm{T}$ cells from ccRCC patients than an equivalent population of $\mathrm{PD}-1^{\text {high }} \mathrm{CD} 8^{+} \mathrm{T}$ cells from healthy donors (Figure 7B).

Given the multiple metabolic impairments in ccRCC CD8 TIL, we tested if rescue of these defective pathways could positively impact TIL activation. Pyruvate was first added to ccRCC T cell stimulation cultures to test if bypassing defects in glucose uptake and glycolysis could restore ccRCC CD8 T cell activation. While no rescue was observed for CD8 TIL from ccRCC patients where TIL activation was partially preserved (Supplemental Figure 9A), provision of pyruvate was sufficient to augment the activation of ccRCC CD8 TIL from patients who otherwise showed poor T cell activation (Figure 8A). SOD2 deficiency can be rescued by provision of SOD2 mimetics and mitochondria-targeted ROS scavengers (48). Treatment with MitoQ to reduce mitochondrial ROS increased the activation of ccRCC CD8 TIL that poorly responded to TCR stimulation (Figure 8B and Supplemental Figure 9B). The mitochondrial ROS scavenger MitoTEMPO treatment also led to increased activation of ccRCC CD8 TIL (Figure 8C). In addition, SOD2 mimetics induced a trend toward increased proliferation and cytokine production by ccRCC 
A

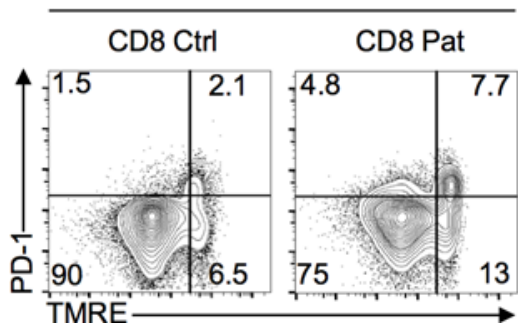

$\mathrm{PB}$

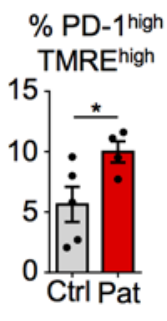

B
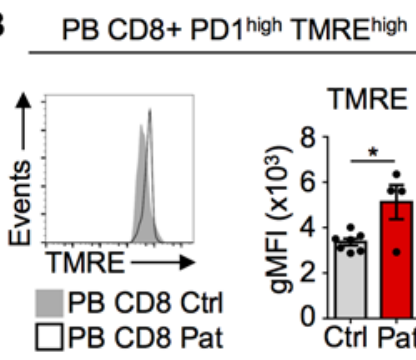

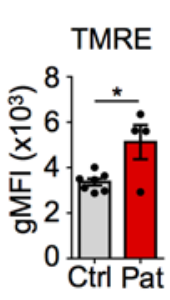

Figure 7. CD8 T cells with signs of mitochondrial dysregulation can be detected in peripheral blood of ccRCC patients. (A and B) Peripheral blood (PB) CD8 T cells from RCC patients $(n=4)$ and healthy controls $(n=5-7)$ were analyzed for PD-1 expression and TMRE accumulation using flow cytometry. Error bars represent $\pm \mathrm{SEM}$; ${ }^{*} P<0.05$, Student's $t$ test.

CD8 TIL (Supplemental Figure 9, C and D). Together, these data support a model in which the ccRCC tumor microenvironment impairs the metabolic status of cCRCC CD8 TIL to directly inhibit activation and that these defects support that these defects may limit the effectiveness of CD8 Teff to mount an immune response to tumor cells (Supplemental Figure 9E).

\section{Discussion}

ccRCC has long held the promise of being an immune-responsive tumor, stemming from a long history of rare but sometimes impressively durable responses to high-dose IL-2, and now, with the enthusiasm around checkpoint-inhibitor therapy, it has been recently approved for kidney cancer (49). Although promising, the response rate of ccRCC to checkpoint therapy is approximately $25 \%$, and durability of response can be limited $(6,7)$, motivating investigations like this to understand the mediators of immune responsiveness or the more common failure of checkpoint therapy to reactivate exhausted T cells in ccRCC. Mechanisms of this therapeutic resistance are poorly understood. It is clear that effector $\mathrm{T}$ cells require metabolic reprogramming for maximal function (50). Here we show, however, that ccRCC CD8 TIL have multiple metabolic impairments that contribute to limited activation. CD8 TIL from ccRCC patients appeared effector-memory-like (31) and were uniformly PD-1 ${ }^{\text {high }}$, which may indicate chronic stimulation and a suppressed or exhausted state. Importantly, ccRCC CD8 TIL had a broad set of defects in glucose uptake, glycolysis, and mitochondrial dynamics and function. These defects contributed to the limited capacity for ccRCC CD8 TIL to activate, as bypassing glycolysis with pyruvate or neutralizing mitochondrial ROS improved markers of $\mathrm{T}$ cell activation. These effects may be related, as pyruvate is also an antioxidant. Metabolic adaptations of TIL to the ccRCC tumor microenvironment may, therefore, form a barrier to antitumor immunity and immune-checkpoint therapy.

Defects in ccRCC CD8 TIL metabolism may arise from several sources. VHL mutations are frequent in ccRCC (28) and lead to a pseudohypoxic response in cancer cells due to stabilization of HIF-1 $\alpha$. This response includes increased aerobic glycolysis and vascularization that may alter the nutrient microenvironment in which $\mathrm{T}$ cells must function. Consumption of intratumoral glucose by cancer cells has been shown to decrease IFN- $\gamma$ production $(10,15,34)$ and may affect the cellular epigenetics through altered
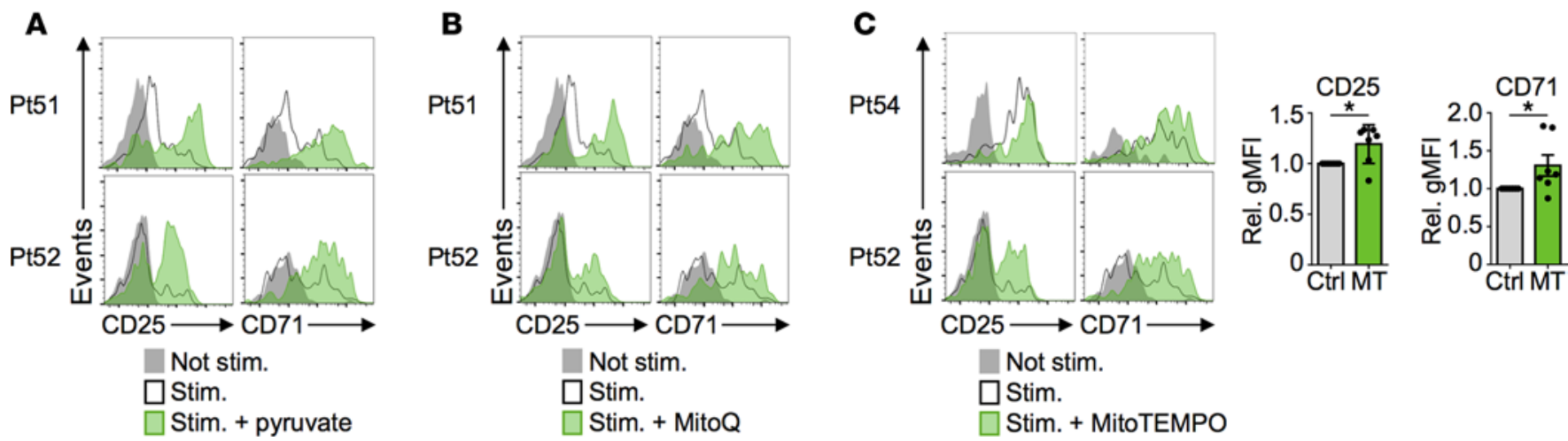

Figure 8. Metabolic rescue increases RCC CD8 TIL activation. (A) RCC patient samples $(n=6)$ were stimulated with or without additional pyruvate ( 5 mM) and activation was measured using CD25 and CD71 expression. Patients were stratified into low responders or high responders based on the activation in the absence of pyruvate supplementation. Data are representative of low-responder patient samples. (B and $\mathbf{C}$ ) Cells were stimulated as in $\mathbf{A}$ and treated with $119 \mathrm{nM}$ MitoQ or $100 \mathrm{nM}$ MitoTEMPO. Expression of CD25 and CD71 was measured with flow cytometry on 6-7 RCC samples. Error bars represent \pm SEM; ${ }^{*} P<0.05$, Student's $t$ test. 
EZH2 expression (25). Here, however, we did not observe a decrease in intratumoral interstitial glucose concentration. This difference may be tumor-type specific or reflect an alternate mechanism by which the tumor microenvironment can influence $\mathrm{T}$ cells. In contrast to glucose, lactate levels were increased, and other metabolites may also be affected. Elevated lactate can impair $\mathrm{T}$ cells $(11,35)$ and may also contribute to T cell metabolic dysfunction. Similarly, PD-1 was elevated on ccRCC CD8 TIL and may impair T cell metabolism. Acute blockade of PD-1 signaling in vitro, however, did not restore ccRCC CD8 TIL function. This may indicate that prolonged PD-1 blockade or additional factors are important for T cells to recover function. Nevertheless, PD-1 can inhibit glycolysis (18-20) and may contribute to poor T cell activation. This effect, however, did not affect all T cells, as CD4 T cells in the same microenvironment were less affected. It is possible that CD4 and CD8 T cells have different nutrient exposures within the tumor microenvironment, or that chronic antigenic stimulation or response to PD-1 signaling differs in these T cell subsets.

Mitochondrial deficiencies appear to play key roles in the inability of TIL to eliminate cancer cells. In contrast to TIL in a melanoma model (26), ccRCC CD8 TIL retained or increased overall mitochondrial mass and appeared to also express PGC1 $\alpha$ and electron transport components at normal levels. Mitochondrial mass was also maintained in TIL from a colorectal carcinoma model, although uncoupling of mitochondrial electron transport was proposed to increase T cell function (51). In ccRCC CD8 TIL, however, mitochondria produced ROS and appeared highly fragmented and in condensed configuration relative to normal healthy CD8 T cell mitochondria. T cell mitochondrial fragmentation can occur upon stimulation (12). Upon activation, fragmentation and cristae remodeling of $\mathrm{T}$ cell mitochondria were associated with decreased electron transfer efficiency and increased glycolysis. Elevated mitochondrial membrane potential and multiple glycolytic defects observed here suggest, however, that ccRCC CD8 TIL have both mitochondrial and glycolytic defects and mitochondrial fragmentation. Chronically altered nutrients or metabolic status can also lead to mitochondrial fragmentation (41) and may contribute to this phenotype in ccRCC CD8 TIL. The extent to which glycolytic and mitochondrial defects may be linked or mediated through distinct mechanisms remains uncertain.

The functional defects of ccRCC CD8 TIL were retained in vitro even when cultured with full nutrient availability. Provision of additional nutrients or treatments to improve metabolic function led to only partial functional rescue. Defects, therefore, were imprinted and long-lasting, and they may reflect an adaptive program to the cCRCC tumor microenvironment. Indeed, ccRCC CD8 TIL did not show a typical impairment of activation when stimulated in low glucose conditions as compared with control $\mathrm{T}$ cells. This adaptive response also likely involves a response to neutralize ROS, as the increase in mitochondrial ROS contrasted with the reduced cytoplasmic redox state. T cells require an increase in cytosolic ROS for calcium signaling upon activation (14), and this may have been blocked by an adaptive response to increased cystine uptake and glutathione production. This adaptive response may have also limited the ability of mitochondrial ROS scavengers to restore ccRCC CD8 T cell function.

The mitochondrial phenotype of ccRCC CD8 TIL may provide a new approach to assess T cell function in ccRCC patients. Tumor-specific T cells have been reported to be observed in the peripheral blood of cancer patients (46), although markers to indicate their functional capacity beyond PD-1 have been lacking. ccRCC CD8 TIL had highly polarized mitochondria, and peripheral blood of ccRCC patients also contained a population of $\mathrm{CD} 8^{+} \mathrm{PD}-1^{+} \mathrm{T}$ cells with elevated mitochondrial polarization. The repertoire and tumor specificity of $\mathrm{CD} 8^{+} \mathrm{PD}-1^{+} \Delta \psi_{\mathrm{m}}{ }^{\text {high }} \mathrm{T}$ cells remains uncertain, but these cells may reflect a population of tumor-experienced lymphocytes. High mitochondrial membrane polarization may mark $\mathrm{T}$ cells with limited antitumor functional capacity. Importantly, tumor-specific CD8 T cells with mitochondrial membrane hyperpolarization expressed inhibitory receptors and had short lifespans and limited ability to control tumors in adoptive T cell therapy studies (39).

The signals and mechanisms that limit $\mathrm{T}$ cell activation and ability to eliminate tumors remain poorly understood. The success of immune therapies, however, demonstrates that $\mathrm{T}$ cells can be reactivated to promote antitumor immunity, albeit currently in a relatively small subset. Maximizing that activity in a broader segment of cancer patients remains the most promising opportunity to be the next major impact in the care of patients in oncology. Improved $\mathrm{T}$ cell metabolism or ability to function in nutrient-poor microenvironments within tumors may be critical. Indeed, PD-1 ligation has been shown to inhibit glycolysis and promote mitochondrial oxidative pathways (18-20), suggesting that a key function of PD-1 blockade may be to improve T cell metabolic fitness. Similarly, chronic stimulation can lead to T cell mitochondrial impairments both in chronic viral infection (20) and melanoma (26). Furthermore, altered mitochondrial 
morphology and membrane hyperpolarization has also been described in HIV-specific CD8 T cells (52) and in systemic lupus erythematosus-associated T cells (53), and normalization of metabolism can reverse autoimmunity (54). Strategies to increase $\mathrm{T}$ cell mitochondrial metabolism in chimeric antigen receptor (CAR) therapy increased in vivo persistence and antitumor responses in human studies (55). Altered mitochondrial dynamics, therefore, may reflect a common response of $\mathrm{T}$ cells to chronic stimulation in a metabolically challenging tumor microenvironment. It is now important to better understand mechanisms that may restrict $\mathrm{T}$ cell metabolism in ccRCC to provide new markers for $\mathrm{T}$ cell function and potential targets to improve $\mathrm{T}$ cell-mediated antitumor immunity.

\section{Methods}

Sample collection and processing. Fresh malignant tissue was surgically removed from 54 patients with RCC. In some cases, adjacent healthy kidney tissue was obtained. Patients with confirmed histology of renal oncocytoma, or papillary or chromophobe RCC were excluded from the study. For certain assays, tissues were placed onto nylon mesh membrane (BioDesign) and centrifuged for 10 minutes at $300 \mathrm{~g}$ to obtain intracellular fluid as described (56). Tissues were processed by mechanical dissociation, followed by enzymatic digestion. Single cell suspension was obtained after passage through a $70-\mu \mathrm{m}$ strainer and subsequent red blood cell lysis. Fresh single cell suspensions were analyzed or in vitro cultured for 3 or 5 days. For mass cytometry experiments, cryopreserved samples were used. PMBC were collected from 7 RCC patients and processed by density gradient centrifugation. Control PBMC were obtained from at least 80 healthy donors.

$T$ cell enrichment, stimulation, and cytokine measurements. RCC tumor single cell suspensions and PBMC from healthy donors and RCC patients were cultured for 3 or 5 days and by addition of anti-CD3 (eBioscience clone UCHT1) in complete RPMI 1640 with $10 \%$ FBS and 1\% penicillin/streptomycin. For selected experiments, cells were cultured in glucose-free RPMI medium with glucose supplementation. In some experiments, cells were stimulated in the presence of anti-PD1 antibody (clone J116, BioXCell). Cells were treated with indicated concentrations of rapamycin (Cell Signaling Technology), metformin (1 mM), phenformin (100 $\mu \mathrm{M}$; both Sigma-Aldrich), MitoQ (MedKoo Biosciences), MitoTEMPO (Sigma-Aldrich), and pyruvate (Sigma-Aldrich). For microscopy experiments, tumor and PBMC single cell suspensions were enriched for CD8 T cells using CD8 microbeads (Miltenyi Biotec). IL-2 and IFN- $\gamma$ production was assessed by Luminex (Thermo Fisher Scientific) assay as described by the manufacturer following stimulation as indicated above and collection of cellular supernatants.

Fluorescence cytometry and mass cytometry. Expression of $\mathrm{T}$ cell surface markers and intracellular targets was measured by fluorescence cytometry (MACSQuant, Miltenyi Biotec) and analyzed by FlowJo software (Tree Star Inc.). The following antibodies were used: anti-CD4 violet blue (VB; clone VIT4), CD8 peridinin chlorophyll (PerCP; clone SK1) or Allophycocyanin (APC), CD71 phycoerythrin (PE; clone OKT9) or APC, CD69 Fluorescein Isothiocyanate (FITC; clone FN50), CD25 PE and APC (clone 4E3), PD-1 APC (clone J105), TIM3 FITC (clone F38-2E2), CTLA4 PE PE (clone 12-1529-41), BTLA FITC (clone ANC5A5), CD200R PE (clone OX108), LAG3 PerCP (clone 3DS223H), CD62L PE-Cy7 (clone DREG56), and CD45R0 APC (clone UCHL1) (all eBioscience, Miltenyi Biotec or BD Biosciences). Staining for intracellular targets was performed after paraformaldehyde fixation and methanol permeabilization using following antibodies: custom made pGlut1 S226 (38), Glut1 (Abcam, clone EPR3915), HK2 (Millipore, polyclonal), pS6 (clone D57.2.2E), GAPDH (clone D16H11), pH2AX (clone 20E3) (all Cell Signaling) and pATM (Millipore, clone 10H11.E12). For SOD1 and SOD2 staining, cells were fixed and permeabilized using the BD Fixation/Permeabilization Kit. For proliferation experiments, cells were stained with Cell Trace Violet dye (Invitrogen), and dye dilution on proliferating cells was measured with fluorescent cytometry. Mass cytometry analyses of tumor infiltrating $\mathrm{T}$ cells were described previously (57). Briefly, cells were stained with cell surface antibodies and subsequently fixed and permeabilized, followed by an overnight incubation with intracellular antibodies. Cells were analyzed on a CyTOF 1.0 at the Vanderbilt Flow Cytometry Shared Resource. Data were bead-normalized and analyzed using Cytobank (58). Expression of 34 markers was used to generate viSNE plots that were utilized to create SPADE analyses.

Microscopy. For immunofluorescent microscopy, cells were isolated as described above and incubated with 300 nM MitoTracker Deep Red (Thermo Fisher Scientific) for 30 minutes, mounted with Wescor Cytopro cytocentrifuge, and then fixed with 4\% PFA (Electron Microscopy Sciences) in cytoskeleton buffer. Cells were permeabilized with 0.1\% Triton X-100 (Sigma-Aldrich), stained for CD8 (Biolegend, 
catalog 344702, clone SK1) and DAPI (300 nM, Thermo Fisher Scientific), and mounted with Prolong Gold Antifade reagent (Thermo Fisher Scientific). Secondary donkey anti-mouse IgG AlexaFluor488 antibodies (Life Technologies, polyclonal) were used. Images were acquired on a Zeiss LSM880 confocal microscope with Airyscan for super-resolution imaging using a $63 \times / 1.40 \mathrm{NA}$ Plan-Apochromat oil objective and 405-, 488-, 561-, and 633-nm lasers and default settings for pinhole and filters. Images were processed using ZEN software and FIJI. To process mitochondrial images in FIJI, 50-pixel rolling ball background subtraction was used. Images of mitochondria are presented as maximum intensity projections or single z-slices for DAPI or CD8. Temporal color code function in FIJI was used for z-series color coding. EM was performed after CD8 $\mathrm{T}$ cell isolation and fixation using a high-resolution transmission electron microscope with eucentric goniometer stage and Gatan cryotransfer stage (Philips/FEI T-12).

$2 N B D G$, cystine uptake, and cysteine detection. Glucose and cystine uptake was measured using the fluorescent glucose analog 2-[N-(7-nitrobenz-2-oxa-1,3-diazol-4-yl)amino]-2-deoxy-D-glucose (2-NBDG) and CystineFITC (44). Cells were cultured in glucose-free media and incubated in $50 \mu \mathrm{M} 2-\mathrm{NBDG}$ for 45 minutes or in $1 \mu \mathrm{M}$ CystineFITC for 20 minutes. After staining of cell surface molecules, fluorescence of selected $\mathrm{T}$ cell subpopulations was measured by flow cytometry. Intracellular cysteine levels were measured as described previously (59) in combination with cell surface markers.

Assessment of mitochondrial morphology and detection of ROS. Mitochondrial membrane potential was measured using TMRE $(0.2 \mu \mathrm{M}$; Thermo Fisher Scientific) according to manufacturer protocols. Mitochondrial mass was assessed using MitoTracker Green, and mitochondrial and cellular ROS were measured using the MitoSOX and DCFDA reagents (all Invitrogen), respectively.

TCGA data processing and IHC. TCGA data were accessed through cBioportal (60). All available cancer types except diseases originating from BM or lymphatic tissue were included. IHC was performed on formalin-fixed, paraffin-embedded RCC tissue samples using DAPI and antibodies against CD8.

Statistics. Data involving 2 groups were analyzed using a paired or unpaired 2-tailed Student $t$ test or Wilcoxon rank sum test with continuity correction. Statistical analysis was performed using Prism (GraphPad Software). For experiments where relative mean fluorescence intensity (MFI) is presented, MFI was normalized to the mean of MFI from controls samples from the same experiment.

Study approval. All subjects (Supplemental Table 1) were deidentified and provided written informed consent according to protocols approved by IRB of the collecting center (University of North Carolina Medical Center and Vanderbilt University Medical Center) in accordance with the Declaration of Helsinki.

\section{Author contributions}

PJS designed and performed the experiments, analyzed the data, and wrote the manuscript. WKR and JCR designed experiments and wrote the manuscript. KEB performed EM experiments. FMM performed super-resolution confocal microscopy. GA preformed NMR experiments. ARG and JMI helped design and perform the mass cytometry experiments. PJS, KEB, ABS, YCJC, and ALC processed RCC tissue. BGV and CLC evaluated data. RCW provided pGlut1 S226 antibody. BK and JH provided expertise concerning the CystineFITC probe. BGV, CLC, TNB and LFG evaluated data.

\section{Acknowledgments}

We thank the patients who participated by donating their tissue for this research and the Cooperative Human Tissue Network Western Division for RCC sample preparation. We also thank the members of WKR and JCR laboratories for support and helpful discussions. This work was supported by the Forbeck Foundation (WKR, BGV), the Cancer Research Institute (JCR, KEB), Brock Fellowship Endowment (KEB), Vanderbilt-Incyte Alliance (JCR, WKR), the German Research Foundation (PJS; DFG KFO 262), and National Cancer Institute K24 CA172355 (WKR). Electron and confocal microscopy was performed in part through the use of the Vanderbilt Cell Imaging Shared Resource (supported by NIH grants CA68485, DK20593, DK58404, DK59637 and EY08126).

Address correspondence to: W. Kimryn Rathmell, 2220 Pierce Avenue, Preston Research Building, Suite 798C, Nashville, Tennessee 37232-2363, USA. Phone: 615.875.9731; Email: kimryn.rathmell@vanderbilt. edu. Or to: Jeffrey Rathmell, 1161 21st Avenue South, A-5301 MCN, Nashville, Tennessee 37232-2363, USA. Phone: 615.936.1764; Email: jeff.rathmell@vanderbilt.edu. 
1. Melero I, et al. Therapeutic vaccines for cancer: an overview of clinical trials. Nat Rev Clin Oncol. 2014;11(9):509-524.

2. Galon J, et al. Type, density, and location of immune cells within human colorectal tumors predict clinical outcome. Science. 2006;313(5795):1960-1964.

3. Galon J, Angell HK, Bedognetti D, Marincola FM. The continuum of cancer immunosurveillance: prognostic, predictive, and mechanistic signatures. Immunity. 2013;39(1):11-26.

4. Sharma P, Allison JP. Immune checkpoint targeting in cancer therapy: toward combination strategies with curative potential. Cell. 2015;161(2):205-214.

5. Topalian SL, Drake CG, Pardoll DM. Immune checkpoint blockade: a common denominator approach to cancer therapy. Cancer Cell. 2015;27(4):450-461.

6. Motzer RJ, et al. Nivolumab versus Everolimus in Advanced Renal-Cell Carcinoma. N Engl J Med. 2015;373(19):1803-1813.

7. Carlo MI, Voss MH, Motzer RJ. Checkpoint inhibitors and other novel immunotherapies for advanced renal cell carcinoma. Nat Rev Urol. 2016;13(7):420-431.

8. Jonasch E, Gao J, Rathmell WK. Renal cell carcinoma. BMJ. 2014;349:g4797.

9. Macintyre AN, et al. The glucose transporter Glut1 is selectively essential for CD4 T cell activation and effector function. Cell Metab. 2014;20(1):61-72.

10. Chang $\mathrm{CH}$, et al. Metabolic Competition in the Tumor Microenvironment Is a Driver of Cancer Progression. Cell. 2015;162(6):1229-1241.

11. Brand A, et al. LDHA-Associated Lactic Acid Production Blunts Tumor Immunosurveillance by T and NK Cells. Cell Metab. 2016;24(5):657-671.

12. Buck MD, et al. Mitochondrial Dynamics Controls T Cell Fate through Metabolic Programming. Cell. 2016;166(1):63-76

13. Cogliati S, et al. Mitochondrial cristae shape determines respiratory chain supercomplexes assembly and respiratory efficiency. Cell. 2013;155(1):160-171.

14. Sena LA, et al. Mitochondria are required for antigen-specific $\mathrm{T}$ cell activation through reactive oxygen species signaling. Immunity. 2013;38(2):225-236.

15. Chang $\mathrm{CH}$, et al. Posttranscriptional control of T cell effector function by aerobic glycolysis. Cell. 2013;153(6):1239-1251.

16. Nakaya $\mathrm{M}$, et al. Inflammatory $\mathrm{T}$ cell responses rely on amino acid transporter ASCT2 facilitation of glutamine uptake and mTORC1 kinase activation. Immunity. 2014;40(5):692-705.

17. Sinclair LV, Rolf J, Emslie E, Shi YB, Taylor PM, Cantrell DA. Control of amino-acid transport by antigen receptors coordinates the metabolic reprogramming essential for T cell differentiation. Nat Immunol. 2013;14(5):500-508.

18. Parry RV, et al. CTLA-4 and PD-1 receptors inhibit T-cell activation by distinct mechanisms. Mol Cell Biol. 2005;25(21):9543-9553.

19. Patsoukis N, et al. PD-1 alters T-cell metabolic reprogramming by inhibiting glycolysis and promoting lipolysis and fatty acid oxidation. Nat Commun. 2015;6:6692.

20. Bengsch B, et al. Bioenergetic Insufficiencies Due to Metabolic Alterations Regulated by the Inhibitory Receptor PD-1 Are an Early Driver of CD8(+) T Cell Exhaustion. Immunity. 2016;45(2):358-373.

21. Patsoukis N, Li L, Sari D, Petkova V, Boussiotis VA. PD-1 increases PTEN phosphatase activity while decreasing PTEN protein stability by inhibiting casein kinase 2. Mol Cell Biol. 2013;33(16):3091-3098.

22. O'Neill LA, Kishton RJ, Rathmell J. A guide to immunometabolism for immunologists. Nat Rev Immunol. 2016;16(9):553-565.

23. Tkachev V, et al. Programmed death-1 controls T cell survival by regulating oxidative metabolism. J Immunol. 2015;194(12):5789-5800.

24. Ho PC, et al. Phosphoenolpyruvate Is a Metabolic Checkpoint of Anti-tumor T Cell Responses. Cell. 2015;162(6):1217-1228.

25. Zhao E, et al. Cancer mediates effector $\mathrm{T}$ cell dysfunction by targeting microRNAs and EZH2 via glycolysis restriction. Nat Immunol. 2016;17(1):95-103.

26. Scharping NE, et al. The Tumor Microenvironment Represses T Cell Mitochondrial Biogenesis to Drive Intratumoral T Cell Metabolic Insufficiency and Dysfunction. Immunity. 2016;45(2):374-388.

27. Siska PJ, et al. Suppression of Glut1 and Glucose Metabolism by Decreased Akt/mTORC1 Signaling Drives T Cell Impairment in B Cell Leukemia. J Immunol. 2016;197(6):2532-2540.

28. Keefe SM, Nathanson KL, Rathmell WK. The molecular biology of renal cell carcinoma. Semin Oncol. 2013;40(4):421-428.

29. Cancer Genome Atlas Research Network. Comprehensive molecular characterization of clear cell renal cell carcinoma. Nature. 2013;499(7456):43-49.

30. Ascierto ML, et al. The Intratumoral Balance between Metabolic and Immunologic Gene Expression Is Associated with AntiPD-1 Response in Patients with Renal Cell Carcinoma. Cancer Immunol Res. 2016;4(9):726-733.

31. Wang QJ, Hanada K, Robbins PF, Li YF, Yang JC. Distinctive features of the differentiated phenotype and infiltration of tumor-reactive lymphocytes in clear cell renal cell carcinoma. Cancer Res. 2012;72(23):6119-6129.

32. Prinz PU, Mendler AN, Masouris I, Durner L, Oberneder R, Noessner E. High DGK- $\alpha$ and disabled MAPK pathways cause dysfunction of human tumor-infiltrating CD8+ T cells that is reversible by pharmacologic intervention. J Immunol. 2012;188(12):5990-6000.

33. Wang $\mathrm{R}$, et al. The transcription factor Myc controls metabolic reprogramming upon T lymphocyte activation. Immunity. 2011;35(6):871-882.

34. Cham CM, Gajewski TF. Glucose availability regulates IFN-gamma production and p70S6 kinase activation in CD8+ effector T cells. J Immunol. 2005;174(8):4670-4677.

35. Haas R, et al. Lactate Regulates Metabolic and Pro-inflammatory Circuits in Control of T Cell Migration and Effector Functions. PLoS Biol. 2015;13(7):e1002202.

36. Buck MD, O'Sullivan D, Pearce EL. T cell metabolism drives immunity. J Exp Med. 2015;212(9):1345-1360.

37. Phan AT, et al. Constitutive Glycolytic Metabolism Supports CD8(+) T Cell Effector Memory Differentiation during Viral Infection. Immunity. 2016;45(5):1024-1037.

38. Lee EE, et al. A Protein Kinase C Phosphorylation Motif in GLUT1 Affects Glucose Transport and is Mutated in GLUT1 Deficiency Syndrome. Mol Cell. 2015;58(5):845-853.

39. Sukumar M, et al. Mitochondrial Membrane Potential Identifies Cells with Enhanced Stemness for Cellular Therapy. Cell Metab. 
2016;23(1):63-76.

40. Youle RJ, van der Bliek AM. Mitochondrial fission, fusion, and stress. Science. 2012;337(6098):1062-1065.

41. Mishra P, Chan DC. Metabolic regulation of mitochondrial dynamics. J Cell Biol. 2016;212(4):379-387.

42. Beier UH, et al. Essential role of mitochondrial energy metabolism in Foxp $3^{+}$T-regulatory cell function and allograft survival. FASEB J. 2015;29(6):2315-2326.

43. Mills EL, et al. Succinate Dehydrogenase Supports Metabolic Repurposing of Mitochondria to Drive Inflammatory Macrophages. Cell. 2016;167(2):457-470.e13.

44. Siska PJ, et al. Fluorescence-based measurement of cystine uptake through $\mathrm{xCT}$ shows requirement for ROS detoxification in activated lymphocytes. J Immunol Methods. 2016;438:51-58.

45. Che M, Wang R, Li X, Wang HY, Zheng XF. Expanding roles of superoxide dismutases in cell regulation and cancer. Drug Discov Today. 2016;21(1):143-149.

46. Cohen CJ, et al. Isolation of neoantigen-specific T cells from tumor and peripheral lymphocytes. J Clin Invest. 2015;125(10):3981-3991.

47. Gros A, et al. Prospective identification of neoantigen-specific lymphocytes in the peripheral blood of melanoma patients. Nat Med. 2016;22(4):433-438

48. Dikalova AE, et al. Therapeutic targeting of mitochondrial superoxide in hypertension. Circ Res. 2010;107(1):106-116.

49. Rini BI, et al. Society for Immunotherapy of Cancer consensus statement on immunotherapy for the treatment of renal cell carcinoma. J Immunother Cancer. 2016;4:81.

50. O'Sullivan D, Pearce EL. Targeting T cell metabolism for therapy. Trends Immunol. 2015;36(2):71-80.

51. Chamoto K, et al. Mitochondrial activation chemicals synergize with surface receptor PD-1 blockade for T cell-dependent antitumor activity. Proc Natl Acad Sci USA. 2017;114(5):E761-E770.

52. Petrovas C, et al. Increased mitochondrial mass characterizes the survival defect of HIV-specific CD8(+) T cells. Blood. 2007;109(6):2505-2513.

53. Perl A, Gergely P, Nagy G, Koncz A, Banki K. Mitochondrial hyperpolarization: a checkpoint of T-cell life, death and autoimmunity. Trends Immunol. 2004;25(7):360-367.

54. Yin Y, et al. Normalization of CD4+ T cell metabolism reverses lupus. Sci Transl Med. 2015;7(274):274ra18.

55. Kawalekar OU, et al. Distinct Signaling of Coreceptors Regulates Specific Metabolism Pathways and Impacts Memory Development in CAR T Cells. Immunity. 2016;44(2):380-390.

56. Haslene-Hox $\mathrm{H}$, et al. A new method for isolation of interstitial fluid from human solid tumors applied to proteomic analysis of ovarian carcinoma tissue. PLoS One. 2011;6(4):e19217.

57. Leelatian N, et al. Single cell analysis of human tissues and solid tumors with mass cytometry. Cytometry B Clin Cytom 2017;92(1):68-78

58. Kotecha N, Krutzik PO, Irish JM. Web-based analysis and publication of flow cytometry experiments. Curr Protoc Cytom. 2010; Chapter 10:Unit10.17.

59. Jung HS, et al. Molecular modulated cysteine-selective fluorescent probe. Biomaterials. 2012;33(33):8495-8502.

60. Cerami E, et al. The cBio cancer genomics portal: an open platform for exploring multidimensional cancer genomics data. Cancer Discov. 2012;2(5):401-404. 